\title{
Proximal Analysis and the Minimal Time Function of a Class of Semilinear Control Systems
}

\author{
Yi Jiang · Yiran He · Jie Sun
}

Received: 3 Decmber 2014. / Accepted: 11 November 2015.

\begin{abstract}
The minimal time function of a class of semilinear control systems is considered in Banach spaces, with the target set being a closed ball. It is shown that the minimal time functions of the Yosida approximation equations converge to the minimal time function of the semilinear control system. Complete characterization is established for the subdifferential of the minimal time function satisfying the Hamilton-Jacobi-Bellman equation. These results extend the theory of finite dimensional linear control systems to infinite dimensional semilinear control systems.
\end{abstract}

Keywords Hamilton-Jacobi-Bellman equation - Minimal time function . Subdifferential · Time optimal control

Mathematics Subject Classification (2010) 93C23 - 90C48 · 49J52

\section{Introduction}

We consider a time optimal control problem in a Banach space (see a detailed definition in Sect. 2 below). The goal of the optimal control problem is to steer an initial point to a given nonempty and closed set along a trajectory of the control system in minimal time. The optimal value function of the optimal control problem is called the minimal time function.

The infinite dimensional semilinear system has been a focal point of research since 1990s; see, e.g., [1-3] and the references therein. An important

Y. Jiang · Y. He

VC/VR Lab and Department of Mathematics, Sichuan Normal University, Chengdu, China.

E-mail: yijiang103@163.com, yiranhe@hotmail.com

$凶$ J. Sun

Department of Decision Sciences, National University of Singapore and Department of Mathematics and Statistics, Curtin University, Bentley, Australia.

E-mail: jie.sun@curtin.edu.au 
topic in this area is the property of the subdifferential of the minimal time function satisfying the Hamilton-Jacobi-Bellman equation. Bardi [4] proved that the minimal time function for general nonlinear control problems is a viscosity solution to the Hamilton-Jacobi-Bellman equation and is the unique viscosity solution of a boundary value problem. Soravia [5] extended Bardi's results to allow noncontrollability assumptions and more general boundary conditions. Recently, Cannarsa and Cârjă [6] showed that the subdifferentials of the minimal time function for the semilinear control system satisfy the Hamilton-Jacobi-Bellman equation by an appropriate Kružkov-type transformation. This result is elegant, but certain important cases are not covered in the analysis. There have been some recent papers addressing the missing cases of [6] such as [7-15], but they are either on simpler systems (constant or linear) or restricted to finite dimensional spaces.

In this paper, we extend the results in [7-15] to semilinear systems in infinite dimensional spaces. We also obtain a complete characterization of the proximal subdifferential of the minimal time function. There are two key difficulties that we have to overcome. One is the unboundedness of the generator of a semigroup. We use the Yosida approximation to guarantee certain regularity. The other is the convergence of the minimal time function of the Yosida approximation equation. By estimating its upper bound and by using the principle of optimality, we establish the desired convergence result.

The organization of the paper is as follows. Section 2 presents basic notions, assumptions, and related results about the time optimal control problem of the semilinear control system. In Sect. 3, we prove that the minimal time functions of the Yosida approximation equations converge to the minimal time function of the semilinear control system. In Sect. 4, we establish a characterization of the subdifferential of the minimal time function satisfying the HamiltonJacobi-Bellman equation. Section 5 concludes this paper.

\section{The Time Optimal Control Problem of the Semilinear Control System}

Let $X$ be a Banach space and consider the time optimal control problem of the semilinear control system

$$
\dot{x}(t)=A x(t)+f(x(t))+u(t) \text { and } x(0)=x_{0},
$$

where $A$ is the generator of a $C_{0}$ semigroup, $f$ is a Lipschitz continuous function, and $u:[0,+\infty[\rightarrow U$ is a measurable function, which is called a control strategy. For some given $M, R>0$, we assume that the set of all control strategies $\mathbb{U}$ is a closed ball $\bar{B}_{M}:=\{x \in X:\|x\| \leqslant M\}$, and the target set $S$ is a closed ball $\bar{B}_{R}:=\{x \in X:\|x\| \leqslant R\}$.

The following basic hypotheses are used in $[3,6]$ and are adopted throughout this paper. 
(H1) $A: D(A) \subset X \rightarrow X$ is the infinitesimal generator of a $C_{0}$ semigroup of bounded linear operators on $\mathrm{X}$, satisfying

$$
\left\|e^{t A}\right\| \leqslant e^{-w t}, \quad \forall t \geqslant 0, \text { for certain constant } w>0 .
$$

(H2) $f: X \rightarrow X$ is a Lipschitz continuous function satisfying

$$
\|f(x)-f(y)\| \leqslant L\|x-y\|, \text { for all } x, y \in X, \text { and } f(0)=0 .
$$

(H3) $M>L R$.

Under (H1) and (H2), for any $x_{0} \in X$ and any control $u$, the mild solution of the semilinear control system (2) uniquely exists. That is, there exists a unique $x\left(t, x_{0}, u\right) \in C([0,+\infty[; X)$ satisfying

$$
x\left(t, x_{0}, u\right)=e^{t A} x_{0}+\int_{0}^{t} e^{(t-s) A}[f(x(s))+u(s)] d s, \forall t \geqslant 0 .
$$

This solution is also called the trajectory of the semilinear control system (2) starting from $x_{0}$ with control $u$, and is often simply denoted by $x(t)$. Assumption (H3) is a controllability condition. We can see its effects in Corollary 3.1. To see that these assumptions are nontrivial, a parabolic state equation in Sobolev spaces is given as an example in Albano et al. [16].

Consider the time optimal control problem for (2). For any control strategy $u \in X$, if $x_{0} \notin S$, we define

$\tau_{\min }:=\min \left\{\tau>0:\right.$ There exists $x\left(t, x_{0}, u\right)$ satisfying $(2)$ and $\left.x\left(\tau, x_{0}, u\right) \in S\right\}$.

For any $x_{0} \in X$ and any control strategy $u \in X$, the transition time function $\theta\left(x_{0}, u\right)$ from $x_{0}$ to $S$ is defined as

$$
\theta\left(x_{0}, u\right):= \begin{cases}\tau_{\min }, & \text { if } x_{0} \notin S \\ 0, & \text { if } x_{0} \in S\end{cases}
$$

The controllable set is given by $\mathfrak{C}:=\left\{x_{0}: \theta\left(x_{0}, u\right)<+\infty\right.$, for some $\left.u\right\}$.

The minimal time function $T: \mathfrak{C} \rightarrow[0,+\infty[$ is defined as

$$
T\left(x_{0}\right):=\inf _{u \in \mathbb{U}} \theta\left(x_{0}, u\right)
$$

The Yosida approximation equation, based on (2), is

$$
\dot{x}_{\mu}(t)=A_{\mu} x(t)+f\left(x_{\mu}(t)\right)+u(t) \text { and } x_{\mu}(0)=x_{0},
$$

where $A_{\mu}=\mu A(\mu-A)^{-1}, \mu>-w$. Under (H2), the unique mild solution $x_{\mu}(t) \in C([0,+\infty[; X)$ of $(7)$ satisfies

$$
\left.x_{\mu}\left(t, x_{0}, u\right)\right)=e^{t A_{\mu}} x_{0}+\int_{0}^{t} e^{(t-s) A_{\mu}}\left[f\left(x_{\mu}(s)\right)+u(s)\right] d s, \forall t \geqslant 0
$$


Then, the transition time function of the Yosida approximation is defined as

$$
\theta_{\mu}\left(x_{0}, u\right):= \begin{cases}\tau_{\mu} & \text { if } x_{0} \notin S ; \\ 0, & \text { if } x_{0} \in S,\end{cases}
$$

where

$\tau_{\mu}:=\min \left\{\tau>0:\right.$ There exists $x_{\mu}\left(t, x_{0}, u\right)$ satisfying $(7)$ and $\left.x_{\mu}\left(\tau, x_{0}, u\right) \in S\right\}$.

The corresponding controllable set is denoted by

$$
\mathfrak{C}_{\mu}:=\left\{x_{0}: \theta_{\mu}\left(x_{0}, u\right)<+\infty, \text { for some } u\right\} .
$$

The minimal time function is then defined as $T_{\mu}\left(x_{0}\right):=\inf _{u \in \mathbb{U}} \theta_{\mu}\left(x_{0}, u\right)$.

One of the main properties of Yosida's approximation can be seen in [3] (p. 68) and [17] (p.376), which is as follows.

Proposition 2.1 Let $A_{\mu}$ be the Yosida approximation of $A, x\left(t, x_{0}, u\right)$ and $x_{\mu}\left(t, x_{0}, u\right)$ be the corresponding mild solutions of (2) and (7), respectively. Then,

$$
\begin{aligned}
& \lim _{\mu \rightarrow+\infty}\left\|A_{\mu} x_{0}-A x_{0}\right\|=0, \quad \forall x_{0} \in D(A), \\
& \lim _{\mu \rightarrow+\infty}\left\|e^{t A_{\mu}} x_{0}-e^{t A} x_{0}\right\|=0, \quad \forall x_{0} \in X, \quad t \in[0, \tilde{T}], \quad \text { and } \\
& \lim _{\mu \rightarrow+\infty} \sup _{t \in[0, \tilde{T}]}\left\|x_{\mu}\left(t, x_{0}, u\right)-x\left(t, x_{0}, u\right)\right\|=0, \text { where } 0<\tilde{T}<+\infty .
\end{aligned}
$$

\section{Convergence Properties of the Minimal Time Function}

The target of this section is to show that the minimal time function of the Yosida approximation equation converges to the minimal time function of the semilinear control system. We start with two lemmas, whose proofs can be found in $[6,18]$.

Lemma 3.1 Assume (H1) and (H2) hold. For any $x_{0} \in X$, there exists a control $\tilde{u}$ such that the trajectory of the semilinear control system (2) satisfies

$$
\left\|x\left(t, x_{0}, \tilde{u}\right)\right\| \leqslant e^{(L-w) t}\left(\left\|x_{0}\right\|-\frac{M}{L}\right)+\frac{M}{L} e^{-w t}, \quad \forall 0 \leqslant t \leqslant \frac{\left\|x_{0}\right\|}{M} .
$$

Lemma 3.2 Assume ( H1) and (H2) hold. For any $x_{0}, y_{0} \in X$ and any control $u$, the mild solution of the semilinear control system (2) satisfies

$$
\left\|x\left(t, x_{0}, u\right)\right\| \leqslant e^{(L-w) t}\left(\left\|x_{0}\right\|+\frac{M}{L-w}\right)-\frac{M}{L-w}
$$

and

$$
\left\|x\left(t, x_{0}, u\right)-x\left(t, y_{0}, u\right)\right\| \leqslant e^{(L-w) t}\left\|x_{0}-y_{0}\right\|, \quad \forall t \in[0,+\infty[.
$$


Remark 3.1 From Lemma 3.2, we can see that, if $\left\|x_{0}\right\| \leqslant \rho$, then $\|f(x(t))\| \leqslant L\|x(t)\| \leqslant C_{\rho}$, for all $t \in[0, T]$, where $0<T<+\infty, \rho>0$, and $C_{\rho}>0$.

Proposition 3.1 Assume (H1)-(H3) hold. Then, the following properties hold

(i) If $L-w>0$, then there exists $\bar{\delta} \in] 0, \frac{M}{L}-R[$ such that

$$
T\left(x_{0}\right) \leqslant \frac{d_{S}\left(x_{0}\right)}{(L-w)\left[\frac{M}{L}-(R+\bar{\delta})\right]}
$$

and

$$
T\left(x_{0}\right) \leqslant \frac{1}{L-w} \log \frac{\frac{M}{L}-R}{\frac{M}{L}-\left\|x_{0}\right\|}<\frac{\left\|x_{0}\right\|}{M},
$$

for all $x_{0}$ satisfying $d_{S}\left(x_{0}\right) \leqslant \bar{\delta}$.

(ii) If $L-w \leqslant 0$, then there exists $\bar{\delta} \in] 0, \frac{M}{L}-R[$ such that

$$
T\left(x_{0}\right) \leqslant \frac{d_{S}\left(x_{0}\right)}{L R}
$$

and

$$
T\left(x_{0}\right) \leqslant \frac{1}{-w} \log \frac{R}{\left\|x_{0}\right\|}<\frac{\left\|x_{0}\right\|}{M},
$$

for all $x_{0}$ satisfying $d_{S}\left(x_{0}\right) \leqslant \bar{\delta}$.

Proof Let $\bar{t}:=\frac{1}{L-w} \log \frac{\frac{M}{L}-R}{\frac{M}{L}-\left\|x_{0}\right\|}$ and $R<\left\|x_{0}\right\|<\frac{M}{L}$. Since

$\lim _{\left\|x_{0}\right\| \rightarrow R}\left(\bar{t}-\frac{\left\|x_{0}\right\|}{M}\right)<0$, there exists a $\left.\bar{\delta} \in\right] 0, \frac{M}{L}-R\left[\right.$ such that $0<d_{S}\left(x_{0}\right)<\bar{\delta}$ and $\bar{t}<\frac{\left\|x_{0}\right\|}{M}$. Now, let $x_{0} \in X$ be fixed so that $0<d_{S}\left(x_{0}\right)<\bar{\delta}$. Lemma 3.1 yields that there exists $\tilde{u}$ satisfying $\left\|x\left(\bar{t}, x_{0}, \tilde{u}\right)\right\| \leqslant R$. Hence, $T\left(x_{0}\right) \leqslant \bar{t}<\frac{\left\|x_{0}\right\|}{M}$. By computation, we can see $\lim _{\left\|x_{0}\right\| \rightarrow R}\left[\left(\frac{M}{L}-R-\bar{\delta}\right)-\left(\frac{\left\|x_{0}\right\|-R}{\frac{M}{L}-R}\right)\right]<0$. Therefore, $T\left(x_{0}\right) \leqslant \bar{t} \leqslant \frac{d_{S}\left(x_{0}\right)}{(L-w)\left[\frac{M}{L}-(R+\bar{\delta})\right]}$.

By the same schemes as above, setting $\bar{t}:=\frac{1}{-w} \log \frac{R}{\left\|x_{0}\right\|}$ and $R<\left\|x_{0}\right\|<\frac{M}{L}$, we can prove (ii).

According to Lemma 3.1 and Proposition 3.1, it is straightforward to have the following corollary related to controllability.

Corollary 3.1 Assume (H1)-(H3) hold and let $\bar{\delta} \in] 0, \frac{M}{L}-R[$. For every $x_{0} \in X$ satisfying $0<d_{S}\left(x_{0}\right) \leqslant \bar{\delta}$, the following properties hold:

(i) If $L-w>0$, then there exists a control $\tilde{u}$ such that the corresponding trajectory $x\left(t, x_{0}, \tilde{u}\right)$ of the semilinear control system (2) over $\left.t \in] 0, \frac{1}{L-w} \log \frac{\frac{M}{L}-R}{\frac{M}{L}-\left\|x_{0}\right\|}\right]$ can reach the target set $S$; 
(ii) If $L-w \leqslant 0$, then there exists a control $\tilde{u}$ such that the corresponding trajectory $x\left(t, x_{0}, \tilde{u}\right)$ of the semilinear control system (2) over $\left.t \in] 0, \frac{1}{-w} \log \frac{R}{\left\|x_{0}\right\|}\right]$ can reach the target set $S$.

Proposition 3.2 Assume $(\mathrm{H} 1)-(\mathrm{H} 3)$ hold and let $x_{0} \in \mathfrak{C} \backslash S$. Then, the minimal time function is locally Lipschizian on $\mathfrak{C} \backslash S$. In other words, there exist $\bar{\sigma}>0$ and $m>0$ such that

$$
\left|T\left(y_{0}\right)-T(z)\right| \leqslant m\left\|y_{0}-z\right\|, \quad \forall y_{0}, z \in B\left(x_{0}, \bar{\sigma}\right) .
$$

Proof First, we consider the case that $L-w>0$. Let $u_{0}$ be a control such that $\theta_{0}:=\theta\left(x_{0}, u_{0}\right)<+\infty$,

$$
\bar{\sigma}:=\min \left\{\bar{\delta} e^{(w-L) \theta_{0}}, \frac{\bar{\delta}}{2} e^{(w-L) \bar{C}}\right\}, \text { and } \bar{C}:=\theta_{0}+\frac{\bar{\delta}}{(L-w)\left[\frac{M}{L}-(R+\bar{\delta})\right]},
$$

where $\bar{\delta} \in] 0, \frac{M}{L}-R\left[\right.$ is the constant in Proposition 3.1. For all $z \in B\left(x_{0}, \bar{\sigma}\right)$, let $y\left(\theta_{0}, z, u_{0}\right)$ be the trajectory of system (2) from $z$ with control $u_{0}$, and $y\left(\theta_{0}, x_{0}, u_{0}\right)$ be the trajectory of system (2) from $x_{0}$ with control $u_{0}$. From Lemma 3.2 , we can see

$$
\begin{aligned}
d_{S}\left(y\left(\theta_{0}, z, u_{0}\right)\right) & \leqslant\left\|y\left(\theta_{0}, z, u_{0}\right)-y\left(\theta_{0}, x_{0}, u_{0}\right)\right\| \\
& \leqslant e^{(L-w) \theta_{0}}\left\|z-x_{0}\right\|<e^{(L-w) \theta_{0}} \bar{\sigma}<\bar{\delta} .
\end{aligned}
$$

When $T(z) \geqslant \theta\left(x_{0}, u_{0}\right)$, Proposition 3.1 and the principle of optimality yield

$$
T(z) \leqslant \theta_{0}+T\left(y\left(\theta_{0}, z, u_{0}\right)\right) \leqslant \theta_{0}+\frac{\bar{\delta}}{(L-w)\left[\frac{M}{L}-(R+\bar{\delta})\right]}=\bar{C} .
$$

When $T(z) \leqslant \theta\left(x_{0}, u_{0}\right)$, it is obvious that $T(z)<\bar{C}$, where $\bar{C}$ is a certain constant. For all $y_{0}, z \in B\left(x_{0}, \bar{\sigma}\right)$, without any loss of generality, we consider $T\left(y_{0}\right)<T(z)$. Hence, for any $\varepsilon \in\left[0, T(z)-T\left(y_{0}\right)\right]$, there exists a control $\hat{u}$ such that $\theta\left(y_{0}, \hat{u}\right)<T\left(y_{0}\right)+\varepsilon<T(z) \leqslant \bar{C}$.

Now, we set $\hat{y}:=y\left(\theta\left(y_{0}, \hat{u}\right), y_{0}, \hat{u}\right)$ and $\hat{z}:=y\left(\theta\left(y_{0}, \hat{u}\right), z, \hat{u}\right)$, which are trajectories of the semilinear control system (2). Since $\hat{y} \in S$, we can obtain

$$
d_{S}(\hat{z}) \leqslant\|\hat{z}-\hat{y}\| \leqslant e^{(L-w) \bar{C}}\left\|y_{0}-z\right\|<2 e^{(L-w) \bar{C}} \bar{\sigma}<\bar{\delta} .
$$

According to Proposition 3.1 and the principle of optimality, we have

$$
\begin{aligned}
\left|T\left(y_{0}\right)-T(z)\right| & \leqslant T(z)-T\left(y_{0}\right) \leqslant T(\hat{z})+\varepsilon \leqslant \frac{\|\hat{z}-\hat{y}\|}{(L-w)\left[\frac{M}{L}-(R+\bar{\delta})\right]}+\varepsilon \\
& \leqslant \frac{e^{(L-w) \bar{C}}\left\|y_{0}-z\right\|}{(L-w)\left[\frac{M}{L}-(R+\bar{\delta})\right]}+\varepsilon .
\end{aligned}
$$

Since $\varepsilon$ is arbitrary, we obtain $\left|T\left(y_{0}\right)-T(z)\right| \leqslant m\left\|y_{0}-z\right\|$, where $m:=\frac{e^{(L-w) \bar{C}}}{(L-w)\left[\frac{M}{L}-(R+\bar{\delta})\right]}$. 
It remains to prove the results when $L-w \leqslant 0$. Let $u_{0}$ be a control such that $\theta_{0}:=\theta\left(x_{0}, u_{0}\right)<+\infty, \bar{\sigma}:=\min \left\{\bar{\delta} e^{(w-L) \theta_{0}}, \frac{\bar{\delta}}{2} e^{(w-L) \bar{C}}\right\}$, and $\bar{C}:=\theta_{0}+\frac{\bar{\delta}}{L R}$, where $\bar{\delta}$ is the constant in Proposition 3.1. Using the same methods as in the case of $L-w>0$, we can obtain that the minimal time function is locally Lipschizian with Lipschitz constant $m=\frac{e^{(L-w)} \bar{C}}{L R}$.

Our next task is to study the properties of the minimal time function of the Yosida approximation equation.

Lemma 3.3 Assume (H1)-(H3) hold. For any $x_{0} \in X$ and $\left.\bar{w} \in\right] 0, w[$, there exist control $\tilde{u}$ and $N>0$ such that when $\mu>N$, the trajectory of control system (7) satisfies

$$
\left\|x_{\mu}\left(t, x_{0}, \tilde{u}\right)\right\| \leqslant e^{(L-\bar{w}) t}\left(\left\|x_{0}\right\|-\frac{M}{L}\right)+\frac{M}{L} e^{-\bar{w} t}, \quad \forall t \in\left[0, \frac{\left\|x_{0}\right\|}{M}\right] .
$$

Proof From Proposition 2.1 and Lemma 3.1, we can see that for any $\varepsilon>0$, there exists $N>0$ such that when $\mu>N$,

$$
\begin{aligned}
\left\|x_{\mu}\left(t, x_{0}, \widetilde{u}\right)\right\| & \leqslant\left\|x_{\mu}\left(t, x_{0}, \widetilde{u}\right)-x\left(t, x_{0}, \widetilde{u}\right)\right\|+\left\|x\left(t, x_{0}, \widetilde{u}\right)\right\| \\
& \leqslant \varepsilon+e^{(L-w) t}\left(\left\|x_{0}\right\|-\frac{M}{L}\right)+\frac{M}{L} e^{-w t}, \quad \forall t \in\left[0, \frac{\left\|x_{0}\right\|}{M}\right] .
\end{aligned}
$$

Let $\varepsilon=e^{(L-\bar{w}) t}\left(\left\|x_{0}\right\|-\frac{M}{L}\right)+\frac{M}{L} e^{-\bar{w} t}-\left[e^{(L-w) t}\left(\left\|x_{0}\right\|-\frac{M}{L}\right)+\frac{M}{L} e^{-w t}\right]$. From $0<\bar{w}<w$, we can see that (18) holds.

By the same arguments as Proposition 3.1, Lemma 3.3 yields the following proposition.

Proposition 3.3 Assume (H1)-(H3) hold. Then, there exists a constant $N>0$ such that for $\mu>N$, the following properties hold.

(i) If $L-\bar{w}>0$, then there exists $\bar{\delta} \in] 0, \frac{M}{L}-R[$ such that

$$
T_{\mu}\left(x_{0}\right) \leqslant \frac{d_{S}\left(x_{0}\right)}{(L-\bar{w})\left[\frac{M}{L}-(R+\bar{\delta})\right]}
$$

and

$$
T_{\mu}\left(x_{0}\right) \leqslant \frac{1}{L-\bar{w}} \log \frac{\frac{M}{L}-R}{\frac{M}{L}-\left\|x_{0}\right\|}<\frac{\left\|x_{0}\right\|}{M},
$$

for all $x_{0}$ satisfying $d_{S}\left(x_{0}\right) \leqslant \bar{\delta}$.

(ii) If $L-\bar{w} \leqslant 0$, then there exists $\bar{\delta} \in] 0, \frac{M}{L}-R[$ such that

$$
T_{\mu}\left(x_{0}\right) \leqslant \frac{d_{S}\left(x_{0}\right)}{L R}
$$

and

$$
T_{\mu}\left(x_{0}\right) \leqslant \frac{1}{-\bar{w}} \log \frac{R}{\left\|x_{0}\right\|}<\frac{\left\|x_{0}\right\|}{M},
$$

for all $x_{0}$ satisfying $d_{S}\left(x_{0}\right) \leqslant \bar{\delta}$. 
Now, we give the convergence properties of the minimal time function.

Theorem 3.1 Assume (H1)-(H3) hold and let $\bar{\delta} \in] 0, \frac{M}{L}-R[$. Then, for every $x_{0} \in X$ satisfying $0<d_{S}\left(x_{0}\right) \leqslant \bar{\delta}$, one has

$$
\lim _{\mu \rightarrow+\infty} T_{\mu}\left(x_{0}\right)=T\left(x_{0}\right) .
$$

Proof The inequalities in Proposition 3.3 tell us that $T_{\mu}\left(x_{0}\right)$ is bounded. Without any loss of generality, we assume

$$
\lim _{\mu \rightarrow+\infty} T_{\mu}\left(x_{0}\right)=T
$$

By definition, there exists a trajectory $y_{\mu}(\cdot)$ of system $(7)$ such that $y_{\mu}\left(T_{\mu}\left(x_{0}\right)\right) \in S$. Applying Proposition 2.1, we can see that $y_{\mu}(\cdot)$ is uniformly convergent to $y(\cdot)$. The equality $(24)$ and the continuity imply $\lim _{\mu \rightarrow+\infty} y_{\mu}\left(T_{\mu}\left(x_{0}\right)\right)=y(T) \in S$. It follows that

$$
T\left(x_{0}\right) \leqslant T=\lim _{\mu \rightarrow+\infty} T_{\mu}\left(x_{0}\right) .
$$

Now we prove the equality holds in (25). If not, then $T\left(x_{0}\right)<\lim _{\mu \rightarrow+\infty} T_{\mu}\left(x_{0}\right)$. It follows for large enough $\mu, T_{\mu}\left(x_{0}\right)>T\left(x_{0}\right)$. Let $\bar{x}(t):=y\left(t, x_{0}, \bar{u}\right)$ and $\bar{u}$ be optimal control so that $\bar{x}\left(T\left(x_{0}\right)\right) \in S$. Proposition 2.1 implies that there exists a trajectory $\bar{y}_{\mu}(t):=y_{\mu}\left(t, x_{0}, \bar{u}\right)$ such that $\lim _{\mu \rightarrow+\infty} \bar{y}_{\mu}(t)=\bar{x}(t)$.

Let $\bar{\delta} \in] 0, \frac{M}{L}-R\left[\right.$ and $0<d_{S}\left(x_{0}\right)<\bar{\delta}$. If $L-\bar{w}>0$, then Lemma 3.3 yields

$$
d_{S}\left(\bar{y}_{\mu}(t)\right) \leqslant\left\|\bar{y}_{\mu}(t)\right\|-R \leqslant e^{(L-\bar{w}) t}\left(\left\|x_{0}\right\|-\frac{M}{L}\right)+\frac{M}{L} e^{-\bar{w} t}-R<\bar{\delta},
$$

for all $t \in\left[0, \frac{1}{L-\bar{w}} \log \frac{\frac{M}{L}-R}{\frac{M}{L}-\left\|x_{0}\right\|}[\right.$. If $L-\bar{w} \leqslant 0$, then Lemma 3.3 yields that

$$
d_{S}\left(\bar{y}_{\mu}(t)\right) \leqslant\left\|\bar{y}_{\mu}(t)\right\|-R \leqslant e^{(L-\bar{w}) t}\left(\left\|x_{0}\right\|-\frac{M}{L}\right)+\frac{M}{L} e^{-\bar{w} t}-R<\bar{\delta},
$$

for all $t \in\left[0, \frac{1}{-\bar{w}} \log \frac{R}{\left\|x_{0}\right\|}[\right.$. From Proposition 3.4 and the principle of optimality, if $L-\bar{w}>0$, then

$$
T_{\mu}\left(x_{0}\right) \leqslant T_{\mu}\left(\bar{y}_{\mu}(t)\right)+t \leqslant \frac{d_{S}\left(\bar{y}_{\mu}(t)\right)}{(L-\bar{w})\left[\frac{M}{L}-(R+\bar{\delta})\right]}+t, \quad \forall t \in\left[0, T_{\mu}\left(x_{0}\right)\right] .
$$

If $L-\bar{w} \leqslant 0$, then

$$
T_{\mu}\left(x_{0}\right) \leqslant T_{\mu}\left(\bar{y}_{\mu}(t)\right)+t \leqslant \frac{d_{S}\left(\bar{y}_{\mu}(t)\right)}{L R}+t, \quad \forall t \in\left[0, T_{\mu}\left(x_{0}\right)\right] .
$$

Letting $\mu \rightarrow+\infty$ on both sides of (26) or (27), we can obtain

$$
\lim _{\mu \rightarrow+\infty} T_{\mu}\left(x_{0}\right) \leqslant \frac{d_{S}(\bar{x}(t))}{(L-\bar{w})\left[\frac{M}{L}-(R+\bar{\delta})\right]}+t, \quad \forall L-\bar{w}>0
$$


and

$$
\lim _{\mu \rightarrow+\infty} T_{\mu}\left(x_{0}\right) \leqslant \frac{d_{\bar{B}_{R}}(\bar{x}(t))}{L R}+t, \quad \forall L-\bar{w} \leqslant 0
$$

Proposition 3.3 implies that $T\left(x_{0}\right)<T_{\mu}\left(x_{0}\right) \leqslant \frac{1}{L-\bar{w}} \log \frac{\frac{M}{L}-R}{\frac{M}{L}-\|x\|}$, for $L-\bar{w}>0$, and $T\left(x_{0}\right)<T_{\mu}\left(x_{0}\right) \leqslant \frac{1}{-\bar{w}} \log \frac{R}{\left\|x_{0}\right\|}$, for $L-\bar{w} \leqslant 0$. Set $t=T\left(x_{0}\right)<T_{\mu}\left(x_{0}\right)$ in (28) and (29); then

$$
T=\lim _{\mu \rightarrow+\infty} T_{\mu}\left(x_{0}\right) \leqslant T\left(x_{0}\right) .
$$

From (25) and (30), we see that the theorem holds.

It should be noted that by Proposition 3.2 and Theorem 3.1, it is easy to see that the minimal time function $T_{\mu}(\cdot)$ is locally Lipschitz on $\mathfrak{C} \backslash S$, as stated in the following proposition.

Proposition 3.4 Assume (H1)-(H3) and given $x_{0} \in \mathfrak{C} \backslash S$. Then, there exists a constant $N>0$. When $\mu>N$, there exist $\bar{\sigma}>0$ and $m>0$ such that

$$
\left|T_{\mu}(y)-T_{\mu}(z)\right| \leqslant m\|y-z\|, \quad \forall y, z \in B\left(x_{0}, \bar{\sigma}\right) .
$$

\section{Proximal Subdifferentials of the Minimal Time Function}

In this section, we present the results for the proximal subdifferentials of the minimal time function that satisfies the Hamilton-Jacobi-Bellman equation.

Let us recall some notions from nonsmooth analysis $[19,20]$. Let $f$ be a proper and lower semicontinuous function with $\operatorname{dom} f:=\{y: f(y)<+\infty\}$. For any $\delta>0$ and $x \in X$, let $B(x, \delta):=\{y \in X:\|x-y\|<\delta\}$.

- The proximal subdifferential of $f$ at $x$ is denoted by $\partial_{P} f(x)$ and is defined as $\xi \in \partial_{P} f(x)$ iff there exist $\sigma>0$ and $\delta>0$ such that $f(x+v)-f(x) \geqslant$ $\langle\xi, v\rangle-\sigma\|v\|^{2}$, for all $v \in B(0, \delta)$.

- The proximal normal cone to a closed set $\Omega$ at $x$ is denoted by $N_{\Omega}^{P}(x)$ and is defined as $\xi \in N_{\Omega}^{P}(x)$ iff there exist $\sigma>0$ and $\eta>0$ such that $\left\langle\xi, s^{\prime}-x\right\rangle \leqslant \sigma\left\|s^{\prime}-x\right\|^{2}$, for all $s^{\prime} \in \Omega \cap B(x, \eta)$. Furthermore, if $\Omega$ is convex, then $\xi \in N_{\Omega}(x)$ satisfies $\left\langle\xi, s^{\prime}-x\right\rangle \leqslant 0$, for all $s^{\prime} \in \Omega$, where $N_{\Omega}(x)$ is the usual normal cone of $\Omega$ at $x$ in the sense of convex analysis.

- The Maximized Hamiltonian function of system (2) is defined as

$$
H(x, \zeta):=\sup _{u \in U}\langle\zeta, A x+f(x)+u\rangle .
$$

- The Hamilton-Jacobi-Bellman Equation of system (2) is defined as $H(x, \zeta)=1$.

Now, we consider that the initial state $x_{0}$ is outside of the target set $S$. For $r \geqslant 0$, define $S(r):=\left\{x_{0} \in X: T\left(x_{0}\right) \leqslant r\right\}$, as the $r$-level set of $T(\cdot)$.

Theorem 4.1 Assume (H1)-(H3) hold. Let $\bar{\delta} \in] 0, \frac{M}{L}-R\left[, x_{0} \in(\mathfrak{C} \backslash S) \cap D(A)\right.$, $0<r:=T\left(x_{0}\right)<+\infty$, and $0<d_{S}\left(x_{0}\right)<\bar{\delta}$. Then, 
(a) $\partial_{P} T\left(x_{0}\right) \subset N_{S(r)}^{P}\left(x_{0}\right) \cap\left\{\xi \in X^{*}: H\left(x_{0},-\xi\right)=1\right\}$;

(b) $\partial_{P} T\left(x_{0}\right)=N_{S(r)}^{P}\left(x_{0}\right) \cap\left\{\xi \in D\left(A^{*}\right): H\left(x_{0},-\xi\right)=1\right\}$.

Proof (a) Let $\xi \in \partial_{P} T\left(x_{0}\right)$. By the definition of proximal subdifferentials, there exist $\sigma>0, \eta>0$ such that for all $y \in B\left(x_{0}, \eta\right)$,

$$
T(y)-T\left(x_{0}\right)-\left\langle\xi, y-x_{0}\right\rangle \geqslant-\sigma\left\|y-x_{0}\right\|^{2} .
$$

It follows that $\left\langle\xi, y-x_{0}\right\rangle \leqslant \sigma\left\|y-x_{0}\right\|^{2}$, for any $y \in S(r) \cap B\left(x_{0}, \eta\right)$, which implies that $\xi \in N_{S(r)}^{P}\left(x_{0}\right)$.

Now, we need to prove $H\left(x_{0},-\xi\right)=1$. For any $\varepsilon>0$, it is clearly that there exists some $v \in U$ such that $\langle\xi, v\rangle \leqslant \inf _{u \in U}\langle\xi, u\rangle+\varepsilon$. Let $u(t)$ be a measurable function satisfying $u(0)=v$. Suppose that $x_{\mu}(t)$ satisfies the following system:

$$
\dot{x}_{\mu}(t)=-A_{\mu} x_{\mu}(t)-f\left(x_{\mu}(t)\right)-u(t) \text { and } x_{\mu}(0)=x_{0},
$$

for all $t \in\left[0,+\infty\left[\right.\right.$. Since $x_{0} \notin S$, for any $\mu>0$, we can find a constant $\lambda>0$ such that $x_{\mu}(t) \notin S$ and $t \in[0, \lambda]$. For $s \in[0, t]$, define $\bar{x}_{\mu}(s):=x_{\mu}(t-s)$ and $\bar{u}(s):=u(t-s)$. Then, $\bar{x}_{\mu}(\cdot)$ is a trajectory of

$$
\dot{\bar{x}}_{\mu}(s)=A_{\mu} \bar{x}_{\mu}(s)+f\left(\bar{x}_{\mu}(s)\right)+\bar{u}(s) \text { and } \bar{x}_{\mu}(0)=x_{\mu}(t) .
$$

By the principle of optimality, we can get

$$
T\left(x_{0}\right)+t=T(x(0))+t=T\left(\bar{x}_{\mu}(t)\right)+t \geqslant T\left(\bar{x}_{\mu}(0)\right)=T\left(x_{\mu}(t)\right) .
$$

It follows from (32) that for $t \in[0, \lambda]$,

$$
t \geqslant T\left(x_{\mu}(t)\right)-T\left(x_{0}\right) \geqslant\left\langle\xi, x_{\mu}(t)-x_{0}\right\rangle-\sigma\left\|x_{\mu}(t)-x_{0}\right\|^{2} .
$$

Dividing both sides of (36) by $t$ and letting $t \rightarrow 0^{+}$, we can see $\left\langle-\xi, A_{\mu} x_{0}+f\left(x_{0}\right)\right\rangle+\sup _{u \in U}\langle-\xi, u\rangle \leqslant 1+\varepsilon$. Let $\mu \rightarrow+\infty$. Then, $\left\langle-\xi, A x_{0}+f\left(x_{0}\right)\right\rangle+\sup _{u \in U}\langle-\xi, u\rangle \leqslant 1+\varepsilon$. Therefore, letting $\varepsilon \rightarrow 0^{+}$, we have

$$
\left\langle-\xi, A x_{0}+f\left(x_{0}\right)\right\rangle+\sup _{u \in U}\langle-\xi, u\rangle \leqslant 1
$$

It remains to show that the equality holds in $(37)$. Let $y(\cdot)$ be an optimal trajectory and $v(\cdot)$ be an optimal control for $T\left(x_{0}\right)$. It follows from the principle of optimality that $T(y(t))+t=T\left(x_{0}\right)$. From (32), note that there exists a constant $\lambda>0$ such that for $t \in[0, \lambda]$,

$$
-t=T(y(t))-T\left(x_{0}\right) \geqslant\left\langle\xi, y(t)-x_{0}\right\rangle-\sigma\left\|y(t)-x_{0}\right\|^{2} .
$$

Dividing both sides of (38) by $t$ and letting $t \rightarrow 0^{+}$, we can obtain

$$
\left\langle\xi, A x_{0}+f\left(x_{0}\right)\right\rangle+\inf _{u \in U}\langle\xi, u\rangle \leqslant\left\langle\xi, A x_{0}+f\left(x_{0}\right)\right\rangle+\langle\xi, v\rangle \leqslant-1 .
$$

Therefore, together with (37), it yields $\left\langle-\xi, A x_{0}+f\left(x_{0}\right)\right\rangle+\sup _{u \in U}\langle-\xi, u\rangle=1$. 
(b) Given (a), we only need to prove

$$
N_{S(r)}^{P}\left(x_{0}\right) \cap\left\{\xi \in D\left(A^{*}\right): H\left(x_{0},-\xi\right)=1\right\} \subset \partial_{P} T\left(x_{0}\right) .
$$

Let $\xi \in N_{S(r)}^{P}\left(x_{0}\right)$ be such that $\left\langle\xi, A x_{0}+f\left(x_{0}\right)\right\rangle+\inf _{u \in U}\langle\xi, u\rangle=-1$. Then, there exist $\sigma_{1}>0, \eta_{1}>0$ such that

$$
\left\langle\xi, y-x_{0}\right\rangle \leqslant \sigma_{1}\left\|y-x_{0}\right\|^{2}, \quad \forall y \in S(r) \cap B\left(x_{0}, \eta_{1}\right)
$$

and $\left\langle\xi, A x_{0}+f\left(x_{0}\right)\right\rangle+\langle\xi, u\rangle \geqslant-1$, for all $u \in U$.

From [3] (p. 53) and $\xi \in D\left(A^{*}\right)$, there exists a constant $\bar{M}>0$ such that

$$
\left\|A_{\mu}^{*} \xi\right\|=\left\|\mu\left(\mu-A^{*}\right)^{-1} A^{*} \xi\right\| \leqslant\left\|\mu\left(\mu-A^{*}\right)^{-1}\right\|\left\|A^{*} \xi\right\| \leqslant \bar{M} .
$$

According to Proposition 2.1 and the Banach-Steinhaus Theorem, we can see that there exists $C>0$ such that $\left\|e^{t A_{\mu}}\right\| \leqslant C$, for $t \in[0, \widehat{T}]$. Let $\varepsilon=1$, it is clearly that there exist constants $k>0$ and $N>0$ such that when $\mu>N$, we can obtain $\left\|\frac{d}{d t}\left(e^{t A_{\mu}} x_{0}\right)\right\|=\left\|e^{t A_{\mu}} A_{\mu} x_{0}\right\| \leqslant C\left(\left\|A x_{0}\right\|+1\right) \leq k$, for all $t \in[0, \widehat{T}]$, where $0<\widehat{T}<+\infty$ and $k$ is the Lipschitz constant of $t \mapsto e^{t A_{\mu}} x_{0}$.

Set

$$
\begin{aligned}
c_{1} & :=m k\left(\eta+\left\|x_{0}\right\|+M+C_{\rho}\right)+1 \text { and } \\
\sigma & :=\min \left\{\sigma_{1} c_{1}^{2}+m[\bar{M}+(L+2 M)\|\xi\|] c_{1}\right\},
\end{aligned}
$$

where $m$ is the Lipschitz constant of the minimal time function $T(\cdot), C_{\rho}$ is the constant in Remark 3.1. Let $\eta:=\min \left\{\bar{\delta}-d_{S}\left(x_{0}\right), \frac{\eta_{1}}{c_{1}}\right\}$ and $\left.\bar{\delta} \in\right] 0, \frac{M}{L}-R[$.

Now, our aim is to prove that for all $y \in B\left(x_{0}, \eta\right)$,

$$
T(y)-T\left(x_{0}\right)-\left\langle\xi, y-x_{0}\right\rangle \geqslant-\sigma\left\|y-x_{0}\right\|^{2} .
$$

That is, $\xi \in \partial_{P} T\left(x_{0}\right)$. If not, then there is $y_{0}$ such that

$$
\left\|y_{0}-x_{0}\right\|<\eta \text { and } T\left(y_{0}\right)-T\left(x_{0}\right)<\left\langle\xi, y_{0}-x_{0}\right\rangle-\sigma\left\|y_{0}-x_{0}\right\|^{2} .
$$

In the following, we divide the discussion into three cases: (1) $T\left(y_{0}\right)=r$, (2) $T\left(y_{0}\right)>r$, and (3) $T\left(y_{0}\right)<r$.

Case (1). If $T\left(y_{0}\right)=r$, then $y_{0} \in S(r)$. Thus (40) contradicts to (42). Hence (41) holds.

Case (2). If $T\left(y_{0}\right)>r$, let $y_{\mu}(t)$ be the optimal trajectory with initial state $y_{0}$ satisfying: $y_{\mu}(t):=e^{t A_{\mu}} y_{0}+\int_{0}^{t} e^{(t-s) A_{\mu}}\left[f\left(y_{\mu}(s)\right)+u(s)\right] d s$, for any $t \in\left[0, T_{\mu}\left(y_{0}\right)\right]$, where $u(t)$ is the optimal control.

By the definition of $\eta$, we can obtain $d_{S}\left(y_{0}\right) \leqslant\left\|y_{0}-x_{0}\right\|+d_{S}\left(x_{0}\right)<\bar{\delta}$. Let $\bar{t}_{\mu}:=T_{\mu}\left(y_{0}\right)-r$. Proposition 3.2 and Theorem 3.1 yield that

$$
\lim _{\mu \rightarrow+\infty} \bar{t}_{\mu}=\bar{t}:=T\left(y_{0}\right)-r>0, \text { and } \lim _{\mu \rightarrow+\infty}\left(\bar{t}_{\mu}-m\left\|y_{0}-x_{0}\right\|\right) \leqslant 0 .
$$

This means that there exists a constant $N>0$. When $\mu>N$, one has

$$
\bar{t}_{\mu}>0, \text { and } \bar{t}_{\mu} \leqslant m\left\|y_{0}-x_{0}\right\| \text {. }
$$


By simple calculus, for all $t \in\left[0, \bar{t}_{\mu}\right]$, we obtain

$$
\begin{aligned}
\left\|y_{\mu}(t)-x_{0}\right\| & \leqslant\left\|e^{t A_{\mu}} y_{0}-x_{0}\right\|+\left\|\int_{0}^{t} e^{(t-s) A_{\mu}}\left[f\left(y_{\mu}(s)\right)+u(s)\right] d s\right\| \\
& \leqslant\left(k \bar{t}_{\mu}+1\right)\left\|y_{0}-x_{0}\right\|+k \bar{t}_{\mu}\left\|x_{0}\right\|+k\left(M+C_{\rho}\right) \bar{t}_{\mu} .
\end{aligned}
$$

When $\mu>N$, for all $t \in\left[0, \bar{t}_{\mu}\right]$, the inequality (43) yields

$$
\left\|y_{\mu}(t)-x_{0}\right\| \leqslant\left[m k\left(\eta+\left\|x_{0}\right\|+M+C_{\rho}\right)+1\right]\left\|y_{0}-x_{0}\right\| \leqslant c_{1}\left\|y_{0}-x_{0}\right\| .
$$

Set $\bar{y}_{\mu}:=y_{\mu}\left(\bar{t}_{\mu}\right)$ for simplification. From (44), we try to estimate formulas to prove $\xi \in \partial_{P} T\left(x_{0}\right)$ as follows

$$
\begin{aligned}
& T\left(y_{0}\right)-r-\left\langle\xi, y_{0}-x_{0}\right\rangle=\bar{t}-\left\langle\xi, y_{0}-\bar{y}_{\mu}+\bar{y}_{\mu}-x_{0}\right\rangle \\
& \geqslant \bar{t}+\int_{0}^{\bar{t}_{\mu}}\left\langle\xi, \dot{y}_{\mu}(s)\right\rangle d s-\left\langle\xi, \bar{y}_{\mu}-x_{0}\right\rangle \\
& \geqslant \bar{t}+\int_{0}^{\bar{t}_{\mu}}\left\langle\xi, \dot{y}_{\mu}(s)-A_{\mu} x_{0}-f\left(x_{0}\right)-u(s)\right\rangle d s-\left\langle\xi, \bar{y}_{\mu}-x_{0}\right\rangle \\
&+\int_{0}^{\bar{t}_{\mu}}\left\langle\xi, A_{\mu} x_{0}+f\left(x_{0}\right)+u(s)\right\rangle d s \\
& \geqslant \bar{t}+\bar{t}_{\mu}\left\langle\xi, A_{\mu} x_{0}+f\left(x_{0}\right)\right\rangle+\int_{0}^{\bar{t}_{\mu}}\langle\xi, u(s)\rangle d s-\left\langle\xi, \bar{y}_{\mu}-x_{0}\right\rangle \\
&+\int_{0}^{\bar{t}_{\mu}}\left(\left\langle\xi, A_{\mu}\left(y_{\mu}(s)-x_{0}\right)\right\rangle+\left\langle\xi, f\left(y_{\mu}(s)\right)-f\left(x_{0}\right)\right\rangle\right) d s \\
& \geqslant \bar{t}+\bar{t}_{\mu}\left\langle\xi, A_{\mu} x_{0}+f\left(x_{0}\right)\right\rangle+\int_{0}^{\bar{t}_{\mu}}\langle\xi, u(s)\rangle d s-\left\langle\xi, \bar{y}_{\mu}-x_{0}\right\rangle \\
&-\int_{0}^{\bar{t}_{\mu}}\left(\left\|A_{\mu}^{*} \xi\right\|\left\|y_{\mu}(s)-x_{0}\right\|+\|\xi\|\left\|f\left(y_{\mu}(s)\right)-f\left(x_{0}\right)\right\|\right) d s \\
& \geqslant \bar{t}+\bar{t}_{\mu}\left\langle\xi, A_{\mu} x_{0}+f\left(x_{0}\right)\right\rangle+\int_{0}^{\bar{t}_{\mu}}\langle\xi, u(s)\rangle d s-m(\bar{M}+L\|\xi\|) c_{1}\left\|y_{0}-x_{0}\right\|^{2} \\
&-\left\langle\xi, \bar{y}_{\mu}-x_{0}\right\rangle .
\end{aligned}
$$

We next estimate the terms in $(45)$. Let $y(t)$ be the trajectory with initial state $y_{0}$ satisfying: $y(t):=e^{t A} y_{0}+\int_{0}^{t} e^{(t-s) A}[f(y(s))+u(s)] d s, \forall t \in\left[0, T\left(y_{0}\right)\right]$. Take $\bar{y}:=y(\bar{t})$. Proposition 2.1 yields that

$$
\lim _{\mu \rightarrow+\infty} \bar{y}_{\mu}=\bar{y} .
$$

From the principle of optimality, we can see $T(y(t))+t=T\left(y_{0}\right)$, for any $t \in\left[0, T\left(y_{0}\right)\right]$. Let $t=\bar{t}$, then $T(y(\bar{t}))=r$. Hence, $y(\bar{t}) \in S(r)$. Moreover, by 
the Lipschitz continuity of $T(\cdot)$, we can get that

$$
\begin{aligned}
\left\|\bar{y}-x_{0}\right\| & \leqslant\left\|e^{\bar{t} A} y_{0}-x_{0}\right\|+\left\|\int_{0}^{\bar{t}} e^{(\bar{t}-s) A}[f(y(s))+u(s)] d s\right\| \\
& \leqslant(k \bar{t}+1)\left\|y_{0}-x_{0}\right\|+k \bar{t}\left\|x_{0}\right\|+k\left(M+C_{\rho}\right) \bar{t} \\
& \leqslant\left[m k\left(\eta+\left\|x_{0}\right\|+M+C_{\rho}\right)+1\right]\left\|y_{0}-x_{0}\right\| \leqslant c_{1}\left\|y_{0}-x_{0}\right\| \leqslant \eta_{1} .
\end{aligned}
$$

This implies $\bar{y} \in S(r) \cap B\left(x_{0}, \eta_{1}\right)$. Letting $\mu \rightarrow+\infty$ in (40), (45) and (46) yields

$$
\begin{aligned}
T\left(y_{0}\right)-r-\left\langle\xi, y_{0}-x_{0}\right\rangle & \left.\geqslant-m c_{1}(\bar{M}+L\|\xi\|)\right]\left\|y_{0}-x_{0}\right\|^{2}-\left\langle\xi, \bar{y}-x_{0}\right\rangle \\
& \geqslant-\left[\sigma_{1} c_{1}^{2}+m c_{1}(\bar{M}+L\|\xi\|)\right]\left\|y_{0}-x_{0}\right\|^{2} \\
& \geqslant-\sigma\left\|y_{0}-x_{0}\right\|^{2} .
\end{aligned}
$$

Then, (47) contradicts (42). Therefore, the result (41) holds.

Case (3). Now we consider $T\left(y_{0}\right)<r$. Consider the trajectory $y_{\mu}(\cdot)$ with initial state $y_{0}$ satisfying $y_{\mu}(t)=e^{-t A_{\mu}} y_{0}-\int_{0}^{t} e^{(s-t) A_{\mu}}[f(y(s))+u(s)] d s$. Let $\bar{t}_{\mu}=r-T_{\mu}\left(y_{0}\right)$. Since $d_{S}\left(y_{0}\right) \leqslant\left\|y_{0}-x_{0}\right\|+d_{S}\left(x_{0}\right)<\bar{\delta}$, Proposition 3.2 yields $\lim _{\mu \rightarrow+\infty} \bar{t}_{\mu}=: \bar{t}=r-T\left(y_{0}\right)>0$ and $\lim _{\mu \rightarrow+\infty}\left(\bar{t}_{\mu}-m\left\|y_{0}-x_{0}\right\|\right) \leq 0$. This means that there exists a constant $N>0$. When $\mu>N$, one has

$$
\bar{t}_{\mu}>0 \text { and } \bar{t}_{\mu} \leqslant m\left\|y_{0}-x_{0}\right\| \text {. }
$$

Let $\bar{y}_{\mu}:=y_{\mu}\left(\bar{t}_{\mu}\right)$. For $t \in\left[0, \bar{t}_{\mu}\right]$, we can get

$$
\begin{aligned}
\left\|y_{\mu}(t)-x_{0}\right\| & \leqslant\left\|e^{t A_{\mu}} y_{0}-x_{0}\right\|+\left\|\int_{0}^{t} e^{(t-s) A_{\mu}}\left[f\left(y_{\mu}(s)\right)+u(s)\right] d s\right\| \\
& \leqslant\left(k \bar{t}_{\mu}+1\right)\left\|y_{0}-x_{0}\right\|+k \bar{t}_{\mu}\left\|x_{0}\right\|+k\left(M+C_{\rho}\right) \bar{t}_{\mu} .
\end{aligned}
$$

When $\mu>N$, for all $t \in\left[0, \bar{t}_{\mu}\right]$, the inequality (48) yields

$$
\left\|y_{\mu}(t)-x_{0}\right\| \leqslant\left[m k\left(\eta+\left\|x_{0}\right\|+M+C_{\rho}\right)+1\right]\left\|y_{0}-x_{0}\right\| \leqslant c_{1}\left\|y_{0}-x_{0}\right\| .
$$

Since $\left\langle\xi, A x_{0}+f\left(x_{0}\right)\right\rangle+\inf _{u \in U}\langle\xi, u\rangle=-1$, for any $\varepsilon>0$, there exists a $v \in U$ such that

$$
\left\langle\xi, A x_{0}+f\left(x_{0}\right)+v\right\rangle \leqslant-1+\varepsilon .
$$

In terms of (49), we next deduce formulas to prove $\xi \in \partial_{P} T\left(x_{0}\right)$.

$$
\begin{aligned}
& T\left(y_{0}\right)-r-\left\langle\xi, y_{0}-x_{0}\right\rangle=-\bar{t}-\left\langle\xi, y_{0}-\bar{y}_{\mu}+\bar{y}_{\mu}-x_{0}\right\rangle \\
& \geqslant-\bar{t}+\int_{0}^{\bar{t}_{\mu}}\left\langle\xi, \dot{y}_{\mu}(s)\right\rangle d s-\left\langle\xi, \bar{y}_{\mu}-x_{0}\right\rangle \\
& \geqslant-\bar{t}+\int_{0}^{\bar{t}_{\mu}}\left\langle\xi, \dot{y}_{\mu}(s)+A_{\mu} x_{0}+f\left(x_{0}\right)+v(s)\right\rangle d s-\left\langle\xi, \bar{y}_{\mu}-x_{0}\right\rangle \\
& -\int_{0}^{\bar{t}_{\mu}}\left\langle\xi, A_{\mu} x_{0}+f\left(x_{0}\right)+v(s)\right\rangle d s \\
& \geqslant-\bar{t}-\bar{t}_{\mu}\left\langle\xi, A_{\mu} x_{0}+f\left(x_{0}\right)\right\rangle-\int_{0}^{\bar{t}_{\mu}}\langle\xi, v(s)\rangle d s-\left\langle\xi, \bar{y}_{\mu}-x_{0}\right\rangle
\end{aligned}
$$




$$
\begin{aligned}
& -\int_{0}^{\bar{t}_{\mu}}\left(\left\langle\xi, A_{\mu}\left(y_{\mu}(s)-x_{0}\right)\right\rangle+\left\langle\xi, f\left(y_{\mu}(s)\right)-f\left(x_{0}\right)+u(s)-v(s)\right\rangle\right) d s \\
& \geqslant-\bar{t}-\bar{t}_{\mu}\left\langle\xi, A_{\mu} x_{0}+f\left(x_{0}\right)\right\rangle-\int_{0}^{\bar{t}_{\mu}}\langle\xi, v(s)\rangle d s-\left\langle\xi, \bar{y}_{\mu}-x_{0}\right\rangle \\
& -\int_{0}^{\bar{t}_{\mu}}\left(\left\|A_{\mu}^{*} \xi\right\|\left\|y_{\mu}(s)-x_{0}\right\|+\|\xi\|\left\|f\left(y_{\mu}(s)\right)-f\left(x_{0}\right)\right\|+2 M\|\xi\|\right) d s \\
& \geqslant-\int_{0}^{\bar{t}_{\mu}}\langle\xi, v(s)\rangle d s-m c_{1}[\bar{M}+(L+2 M)\|\xi\|]\left\|y_{0}-x_{0}\right\|^{2}-\left\langle\xi, \bar{y}_{\mu}-x_{0}\right\rangle \\
& -\bar{t}-\bar{t}_{\mu}\left\langle\xi, A_{\mu} x_{0}+f\left(x_{0}\right)\right\rangle .
\end{aligned}
$$

We analyze the terms in (51). Let $y(t)$ be the trajectory with initial state $y_{0}$ satisfying $y(t):=e^{-t A} y_{0}-\int_{0}^{t} e^{-(t-s) A}[f(y(s))+u(s)] d s$, for all $t \in\left[0, T\left(y_{0}\right)\right]$. Take $\bar{y}:=y(\bar{t})$. Proposition 2.1 yields

$$
\lim _{\mu \rightarrow+\infty} \bar{y}_{\mu}=\bar{y}
$$

If $\bar{y} \notin \bar{B}_{R}$, define $x(s):=y(\bar{t}-s)$ and $g(s):=u(\bar{t}-s)$, for $s \in[0, \bar{t}]$. Then, $x(\cdot)$ is the mild solution satisfying $\dot{x}(s)=A x(s)+f(x(s))+g(s)$ and $x(0)=\bar{y}$. From the principle of optimality, we can see

$$
T(x(0))=T(\bar{y}) \leqslant T(x(s))+s, \quad \forall t \in[0, T(\bar{y})] .
$$

If $\bar{t} \leqslant T(\bar{y})$, take $s=\bar{t}$ in (53), then $T(\bar{y}) \leqslant r$. If not, it is obvious that $T(\bar{y}) \leqslant r$. Hence, $\bar{y} \in S(r)$. By simple calculus, for all $s \in[0, \bar{t}]$, we can obtain

$$
\begin{aligned}
\left\|\bar{y}-x_{0}\right\| & \leqslant\left\|e^{-\bar{t} A} y_{0}-x_{0}\right\|+\left\|\int_{0}^{\bar{t}} e^{(s-\bar{t}) A}[f(y(s))+u(s)] d s\right\| \\
& \leqslant(k \bar{t}+1)\left\|y_{0}-x_{0}\right\|+k \bar{t}\left\|x_{0}\right\|+k\left(M+C_{\rho}\right) \bar{t} \\
& \leqslant\left[m k\left(\eta+\left\|x_{0}\right\|+M+C_{\rho}\right)+1\right]\left\|y_{0}-x_{0}\right\| . \\
& \leqslant c_{1}\left\|y_{0}-x_{0}\right\| \leqslant \eta_{1} .
\end{aligned}
$$

This implies $\bar{y} \in S(r) \cap B\left(x_{0}, \eta_{1}\right)$. Letting $\mu \rightarrow+\infty$ and $\varepsilon \rightarrow 0^{+}$in (40), (50)(52), and (54) yield

$$
\begin{aligned}
T\left(y_{0}\right)-r-\left\langle\xi, y_{0}-x_{0}\right\rangle & \geqslant-m[\bar{M}+(L+2 M)\|\xi\|] c_{1}\left\|y_{0}-x_{0}\right\|^{2}-\left\langle\xi, \bar{y}-x_{0}\right\rangle \\
& \geqslant-\left[\sigma_{1} c_{1}^{2}+m c_{1}(\bar{M}+(L+2 M)\|\xi\|)\right]\left\|y_{0}-x_{0}\right\|^{2} \\
& \geqslant-\sigma\left\|y_{0}-x_{0}\right\|^{2} .
\end{aligned}
$$

Then, (55) contradicts (42). Thus, (41) holds and the proof is completed.

For the case in which the initial state $x_{0}$ is inside of the target set $S$, the proof is similar. For brevity, we only state the result and omit its proof.

Theorem 4.2 Assume (H1)-(H3) hold and let $x_{0} \in S \cap D(A)$. Then,

(a) $\partial_{P} T\left(x_{0}\right) \subset N_{S}\left(x_{0}\right) \cap\left\{\xi \in X^{*}: H\left(x_{0},-\xi\right) \leqslant 1\right\}$;

(b) $\partial_{P} T\left(x_{0}\right)=N_{S}\left(x_{0}\right) \cap\left\{\xi \in D\left(A^{*}\right): H\left(x_{0},-\xi\right) \leqslant 1\right\}$. 


\section{Conclusions}

This paper studies the minimal time function of a semilinear control system with the target set being a closed ball in Banach spaces. We show that the minimal time functions of the Yosida approximation systems converge to the minimal time function of the semilinear control system. We also give a complete characterization for the proximal subdifferential of the minimal time function satisfying the Hamilton-Jacobi-Bellman equation. We therefore establish new results for semilinear control systems in infinite dimensional spaces, which extend the corresponding results in the literature on linear control systems in finite dimensional spaces.

Acknowledgements This work was partially supported by National Natural Science Foundation of China (11201324), Fok Ying Tuny Education Foundation (141114), Outstanding Youth Academic Technology Leader Training Plan of Sichuan province (2013JQ0027), the Provost's Chair Fund of National University of Singapore, and a grant from Faculty of Science and Engineering, Curtin University.

\section{References}

1. Bardi, M., Cannatsa-Dolcetta, I.: Optimal Control and Viscosity Solutions of HamiltonJacobi-Bellman Equations. Birkhäuser Boston, Cambridge (1997)

2. Fattorini, H.O.: Infinite Dimensional Optimization and Control Theory. Cambridge University Press, New York (1996)

3. Li, X.J., Yong, J.M.: Optimal Control Theory for Infinite Dimensional Systems. Birkhäuser Boston, Cambridge (1995)

4. Bardi, M.: A boundary value problem for the minimal time problem. SIAM J. Control Optim. 27, 776-785 (1989)

5. Soravia, P.: Discontinuous viscosity solutions to Dirichlet problems for Hamilton-Jacobi equations with convex Hamiltonians. Comm. Partial Differ. Equ. 18, 1493-1514 (1993)

6. Cannarsa, P., Cârjă, O.: On the Bellman equation for the minimum time problem in infinite dimensions. SIAM J. Control Optim. 43, 532-548 (2004)

7. Wolenski, P.R., Yu, Z.: Proximal analysis and the minimal time function. SIAM J. Control Optim. 36, 1048-1072 (1998)

8. Colombo, G., Wolenski, P.R.: The subgradient formula for the minimal time function in the case of constant dynamics in Hilbert space. J. Glob. Optim. 28, 269-282 (2004)

9. Colombo, G., Wolenski, P.R.: Variational analysis for a class of minimal time functions in Hilbert spaces. J. Convex. Anal. 11, 335-361 (2004)

10. He, Y.R., Ng, K.F.: Subdifferentials of a minimum time function in Banach spaces. J. Math. Anal. Appl. 321, 896-910 (2006)

11. Jiang, Y., He, Y.R.: Subdifferentials of a minimum time function in normed spaces. J. Math. Anal. Appl. 358, 410-418 (2009)

12. Jiang, Y., He, Y.R.: Subdifferential properties for a class of minimal time functions with moving target sets in normed spaces. Appl. Anal. 91, 491-502 (2012)

13. Mordukhovich, B.S., Nguyen, M.N.: Limiting subgradients of minimal time functions in Banach spaces. J. Global Optim. 46, 615-633 (2009)

14. Mordukhovich, B.S., Nguyen, M.N.: Subgradients of minimal time functions under minimal requirements. J. Convex Anal. 18, 915-947 (2011)

15. Jiang, Y., He, Y.R., Sun, J.: Subdifferential properties of the minimal time function of linear control systems. J. Global Optim. 51, 395-412 (2011)

16. Albano, P., Cannarsa, P., Sinestrari, C.: Regularity results for the minimum time function of a class of semilinear evolution equations of parabolic type. SIAM J. Control Optim. 38, 916-946 (2000) 
17. Rudin, W.: Functional Analysis. McGraw-Hill Book Inc, New York (1991)

18. Cârjă, O.: On the minimum time function and the minimum energy problem a nonlinear case. Syst. Control Lett. 55, 543-548 (2006)

19. Clarke, F.H.: Optimization and Nonsmooth Analysis. Wiley, New York (1983)

20. Mordukhovich, B.S.: Variational Analysis and Generalized Differentiation I. Springer, Berlin (2006) 